\section{Danish bispecifics meet ADCs}

Genmab and AbbVie will jointly develop and market up to seven anticancer antibody-based therapeutics, in a deal worth $\$ 750$ million in up-front payments and up to $\$ 3.15$ billion in potential milestone payments. The pact will bring together Genmab's antibodies with AbbVie's antibody-drug conjugate (ADC) technology, which uses antibodies to deliver toxic payloads to cancer cells while sparing non-cancer cells.

With this deal, AbbVie is buying into three of the Copenhagen-based biotech's extant bispecific antibodies, an increasingly important therapeutic class. Genmab's now partnered epcoritamab (DuoBody-CD3xCD20) and DuoBody-CD3x5T4 are T-cell-engaging therapeutics that direct cancer-killing T cells to cancer antigens of interest. Epcoritamab, a phase $1 / 2$ candidate, binds CD3 on $T$ cells and the cancer target CD20 on cancer cells for the treatment of B cell malignancies. DuoBody-CD3x5T4, a preclinical candidate, similarly recruits T cells through CD3 but binds the 5T4 cancer antigen on solid tumor cells. DuoHexaBody-CD37, by contrast, binds two separate sites on the hematological cancer antigen CD37. Rather than driving $T$ cell engagement directly, this HexaBody approach relies on the formation of antibody clusters at the target, enhancing immune effector functions such as complement-mediated cell killing.

The partners will also work together to select and develop up to four other antibody-based candidates, against either solid or hematological cancer targets. AbbVie lists five ADCs in its clinical pipeline. AbbVie hopes any successes will fill an upcoming revenue gap from its anti-tumor necrosis factor- $\alpha$ antibody Humira (adalimumab), a \$15-billion-per-year blockbuster that is set to face biosimilar competition in the United States in 2023.

Genmab's other development partners include Johnson \& Johnson's Janssen Biotech, Roche and Seattle Genetics.

Published online: 8 July 2020 https://doi.org/10.1038/s41587-020-0607-z bind and neutralize the virus, either by blocking spike binding to its receptor or by preventing fusion of the virus with the cell. But if antibodies fail to do this because they bind unproductively to the virus, these non-neutralizing antibodies may instead, using the Fc region at the base of the antibody, latch onto host macrophage Fc receptors and ferry the virus into these host cells, at least in vitro. Once inside, the viruses replicate and then exit to further spread infection to neighboring cells and tissues.

Poor-quality antibodies that bind the virus without neutralizing it are one reason vaccine candidates fail, and, in theory, could also cause ADE, delivering virions to host cells.

Can SARS-CoV-2 infect macrophages? "I don't know," answers Stan Perlman, a coronavirus expert at the University of Iowa. "Those data are not out there yet." The virus can infect macrophages in vitro, but that doesn't necessarily translate to in vivo human biology. "In tissue culture cells you can make almost any virus show this, by jigging the right cells, the right amount of antibody, and the right virus. But that [is] not really meaningful, because of the fact that it's so artificial."

The risk that a COVID-19 vaccine will cause ADE and facilitate infection "is completely theoretical," says Novavax's Glenn. "It's a lab, an animal phenomenon that's been observed. [But] in RSV it's a real clinical event." In a 1960s vaccine trial, $80 \%$ of infants and young children immunized against RSV (respiratory syncytial virus, an RNA virus in the family Paramyxoviridae) ended up hospitalized, and 2 children died. Only $5 \%$ of the children in the control group required hospitalization. Vaccinated children carried high titers of non-neutralizing antibodies, implicating ADE in the tragic outcome, although a $\mathrm{T}_{\mathrm{H}} 2$ (inflammatory) $\mathrm{T}$ cell response also probably contributed.

Since then, some vaccine studies in animals, as well as human trials where vaccinated people had worse outcomes, suggest ADE at work. But definitive evidence is lacking. "We can't say they were not due to ADE, but there's not evidence that they were," says Ahmed. "There are many other ways that an immune response can cause damage."

For coronavirus vaccines, the ADE story is similarly inconclusive. Cats vaccinated with spike protein against feline coronavirus died much faster than unvaccinated cats and carried more anti-spike antibodies, implicating ADE. But macaques vaccinated against SARS-CoV-1 did not show enhanced infection or disease.

In humans, limited data for vaccines against earlier coronaviruses did not show
ADE. Two vaccines against the closely related SARS-CoV-1 appeared safe in early trials. A DNA vaccine against the MERS coronavirus in macaques protected all eight monkeys from infection symptoms, and that MERS vaccine was well-tolerated in a 2016 phase 1 human trial. MERS vaccine co-developer David Weiner, director of the Vaccine \& Immunotherapy Center at the Wistar Institute, co-developed a very similar COVID-19 vaccine that Inovio Pharmaceuticals took into human trials in early April.

"We don't see it [ADE] with flu, we don't see it with so many other vaccines that we have," says Ahmed. "We shouldn't dismiss it completely, but then if you look at the broader world of vaccinology, this has not been seen." But Gilligan says some earlier SARS and MERS vaccine candidates didn't advance because of signs of ADE.

$\mathrm{ADE}$ is a concern for monoclonal antibodies (mAbs) too, at least in theory, because $m A b s$ that fail to neutralize the virus also could facilitate viral entry into cells. Many companies are working on such mAbs for either COVID-19 prevention or therapy. AstraZeneca, for example, is partnering with Vanderbilt, which has identified, expressed and evaluated virus-neutralizing antibodies from B cells recovered from convalescents. AstraZeneca will take the best ones forward into clinical trials.

$\mathrm{ADE}$ is part of the mAb discussion. "There's not a lot of obvious epidemiologic evidence for it yet," says Vanderbilt's Crowe. Nevertheless, he says, "we will address this." Crowe's group will test for ADE by seeing whether its monoclonal antibodies can deliver viruses into macrophages, compared with antibodies whose Fc region has been inactivated. (An antibody lacking Fc can't bind macrophage Fc receptors and thus can't cause ADE. That's why some companies are advancing $\mathrm{mAbs}$ with the $\mathrm{Fc}$ region knocked out.) Still, "the animal models are so inadequate right now, it would be very difficult to prove whether [ADE] is common or uncommon," Crowe says.

For mAbs, the risk is probably low. "You're already characterizing it as a highly neutralizing antibody," says Ahmed. Convalescent antibody passive immunization trials, now underway, could carry some ADE risk, Ahmed says. "[But] I would be less concerned with that than with the vaccine that might be inducing suboptimal response."

Vaccine companies are fully aware of ADE. "All the groups have been discussing this, everyone's on the same page, everyone wants a safe [COVID-19] vaccine," says Weiner. Inovio went forward into the clinic after showing safety in primates, and is 